Unfallchirurg $2010 \cdot 113: 434-435$

DOI 10.1007/s00113-010-1741-8

Online publiziert: 28. Mai 2010

(c) Springer-Verlag 2010

\author{
S.R. Schwarzkopf ${ }^{1} \cdot$ C. Krettek ${ }^{2}$ \\ ${ }^{1}$ Klinik und Poliklinik für Physikalische Medizin und Rehabilitation, \\ Klinikum der Ludwig-Maximilians-Universität München \\ ${ }^{2}$ Unfallchirurgische Klinik, Medizinische Hochschule Hannover
}

\title{
Outcomemessung nach Trauma
}

horte nach mehr als 10 Jahren zeigen, dass die Ursache für die dann weiterhin bestehenden physischen Einschränkungen v. a. im Bereich des Schädels (40\%) im Vordergrund standen, gefolgt von den unteren Extremitäten (34\%) und der Wirbelsäule (15\%). Das Rehabilitationsergebnis wurde von den Patienten in 33\% der Fälle mit gut, in $14,1 \%$ mit sehr gut, in $29,3 \%$ mit zufrieden stellend, in $16 \%$ mit schlecht und in $7,5 \%$ mit unbefriedigend angegeben [5]. Dies spiegelt die beeinträchtigte posttraumatische Funktionsfähigkeit und Überlebensqualität nur partiell wider. Mit der Fokussierung auf die Funktionsfähigkeit als das zentrale medizinische Thema für den Patienten und für die Gesellschaft und auf die posttraumatische Überlebensqualität wurde mittlerweile das Konzept eines umfassenden Outcomeassessments unter Berücksichtigung aller wichtigen Aspekte der persönlichen Krankheitslast des Traumapatienten notwendig $[1,2,4]$.

Es gibt eine Vielzahl von Outcomeinstrumenten und -tests für die Beeinträchtigung spezieller anatomischer Regionen, für die Verletzungsschwere und für physiologische, körperliche und muskuloskelettale Funktionen, für spezielle Versorgungssituationen, für die Einschätzung psychologischer und kognitiver Aspekte und für die Beurteilung der Lebensqualität und der allgemeinen Gesundheit.

Im Rahmen einer großen Studie zeigte sich, dass die primären Verletzungen anatomisch an den unteren Extremitäten, gefolgt von den oberen Extremitäten und an dritter Stelle im Schädelbereich vorlagen. Langzeituntersuchungen dieser Ko-

\section{Die ICF kann zur Klassifizierung aller Aspekte der Funktionsfähigkeit berufsgruppenübergreifend verwendet werden}

Mit der Internationalen Klassifikation der Funktionsfähigkeit, Behinderung und Gesundheit (ICF) steht eine strukturierte Klassifikation zur Verfügung, die alle wichtigen Aspekte des Traumapatienten frührehabilitativen und rehabilitativen Phase jeweils individuell und umfassend darstellen kann. Die ICF wurde im Mai 2001 von der WHO verabschiedet und ist eine Weiterentwicklung der Internationalen Klassifikation von Impairments, Disabilities and Handicaps (ICIDH 1980), von einer Klassifikation der „Krankheitsfolponenten der Gesundheit“. Das der ICF zugrunde liegende biopsychosoziale Modell beinhaltet die verschiedenen Komponenten der Funktionsfähigkeit (Körzu Beginn der Versorgung, in der akuten, gen“ hin zu einer Klassifikation der „Kom- perfunktionen und -strukturen, Aktivitäten und Partizipation) und der Kontextfaktoren (Umwelt- und personbezogene Faktoren).

Als standardisierte internationale Sprache und Dokumentation kann sie berufsgruppenübergreifend und zur Klassifizierung aller Aspekte der Funktionsfähigkeit bei allen Gesundheitsproblemen auf der Ebene des Individuums bis hin zu Bevölkerungsgruppen kulturübergreifend verwendet werden. Die einzelnen traumabedingten Beeinträchtigungen der Funktionsfähigkeit können mit den ICF-Kategorien und den bisher entwickelten ICF Core Sets strukturiert erfasst werden. Mit den ICF-Kategorien können die Inhalte bestehender Instrumente und Skalen auf fehlende Aspekte überprüft und, basierend auf den Kategorien der ICF Core Sets, entsprechende Instrumente entwickelt werden [3]. Einen problemorientierten Ansatz zur multiprofessionellen Therapiekonzeption und Outcomeevaluation stellt der ICF-basierte Rehab-CYCLE dar.

Die demographische Entwicklung mit einer zunehmenden Alterung der Bevölkerung der Industrienationen führt zu einer steigenden Anzahl von Unfällen und Verletzungen im höheren Alter. So weist z. B. der ältere Polytraumapatient andere Verletzungsarten, ein anderes Outcome und einen anderen Therapiebedarf im Vergleich zum erwachsenen Normalkollektiv auf [6].

Unter Berücksichtigung dieser Situation sollte sich der zukünftige kurz- und insbesondere langfristige Outcomefokus auch auf die posttraumatische Funktionsfähigkeit und Überlebensqualität un- 
ter Berücksichtigung der beruflichen und sozialen Integration beziehen. Die umfassende Versorgung von Traumapatienten der verschiedenen Altersgruppen verpflichtet zu einer stetigen Optimierung eines strukturierten Versorgungs- und Outcomemanagements unter Berücksichtigung der Qualitätssicherung, der optimalen Ressourcenallokation und zunehmenden gesundheitsökonomischen $\mathrm{Be}$ lastung unseres Gesundheitssystems und damit unserer Gesellschaft.

Outcomemessung nach Trauma stellt ein zentrales Thema in der Unfallchirurgie dar und ist das Leitthema in der aktuellen Ausgabe von „Der Unfallchirurg“. Die vorliegenden Beiträge sollen die ICF mit ihrer Struktur und dem zugrunde liegenden biopsychosozialen Modell als Klassifikation für die umfassende Darstellung der Funktionsfähigkeit und Behinderung und für die Outcomeevaluation aufzeigen. Die praktische Umsetzung der ICF mit dem Rehab-CYCLE zur Outcomeevaluation wird an einem Patientenbeispiel in einem weiteren Artikel dargestellt. In einem systematischen Review sind die bisher häufig eingesetzten Messinstrumente und ihre Inhalte zur Beurteilung der Funktionsfähigkeit und des funktionalen Outcomes nach Polytrauma aufgeführt und soweit möglich mit den entsprechenden ICF-Kategorien kodiert. Die gesundheitsbezogene Lebensqualität als Outcomekriterium in der Traumatologie wird mit den verfügbaren Instrumenten und Verfahren aus methodischer Sicht und mit entsprechenden Anwendungsbeispielen dargestellt. Die Thematisierung des Outcomes in der Alterstraumatologie wird in einem weiteren Artikel vorgenommen.

Ihre
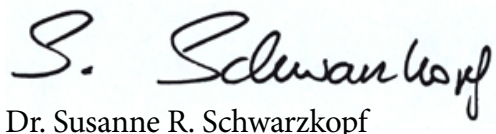

Dr. Susanne R. Schwarzkopf

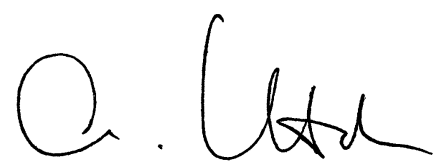

Prof. Dr. Christian Krettek

\section{Korrespondenzadressen}

\section{Dr. S.R. Schwarzkopf}

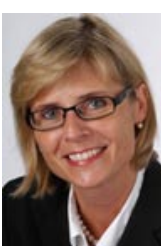

Klinik und Poliklinik

für Physikalische Medizin

und Rehabilitation,

Klinikum der Ludwig-Maximilians-

Universität München,

Marchioninistr. 15,

81377 München

susanne.schwarzkopf@med.

uni-muenchen.de

\section{Prof. Dr. C. Krettek FRACS, FRCSEd}

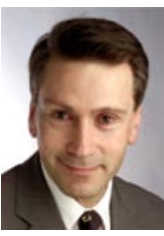

Unfallchirurgische Klinik, Medizinische Hochschule Hannover

Carl-Neuberg-Straße 1, 30625 Hannover

krettek.christian@ mh-hannover.de

\section{Literatur}

1. Bouillon B, Neugebauer E (1998) Outcome after polytrauma. Langenbecks Arch Surg 383:228-234

2. Greenfield $S$ (1989) The state of outcome research: we are on target? N Engl J Med 320:1142-1143

3. Grill E, Stucki G (2009) Scales could be developed based on simple clinical ratings of ICF Core Set categories. J Clin Epidemiol (in press)

4. Neugebauer $E$ (2002) Quality of life after multiple trauma. Restor Neurol Neurosci 20:83-84

5. Pape HC, Zelle B, Lohse R et al (2006) Evaluation and outcome of patients after polytrauma - can patients be recruited for long-term follow-up? Int J Care Injur 37:1197-1203

6. Wutzler S, Lefering R, Laurer HL et al (2008) Veränderungen in der Alterstraumatologie. Eine Analyse an 14.869 Patienten aus dem deutschen Traumaregister. Unfallchirurg 111:592-598

\section{Klinisches Register „RaFTinG" zur Optimierung der Flüssig- keits- und Volumentherapie auf deutschen Intensivstationen}

Unter der Leitung von Prof. Hugo Van Aken (Universitätsklinikum Münster) und Prof. Bernhard Zwißler (Klinikum der Universität München) wird bis zum 28.2.2011 das prospektive klinische Register RaFTinG (Rational Fluid Therapy in Germany, Trial Registration Number NCT01122277) zur Dokumentation der aktuellen infusionstherapeutischen Praxis auf deutschen Intensivstationen durchgeführt. 90 Tage nach Entlassung werden durch ein Follow-up auch Daten in anonymisierter Form zum Langzeit-Outcome erhoben, welche von den teilnehmenden Kliniken zur internen Qualitätskontrolle genutzt werden können. Die Datenerfassung erfolgt internet-basiert direkt auf Station. Zur Mitwirkung sind alle deutschen Kliniken mit Intensivstation eingeladen.

Weitere Informationen erhalten Sie unter www.rafting-register.de. 